\title{
Analysis of Vehicle Platform Vibration Based on Empirical Mode Decomposition
}

\author{
Chengwu Shen $\mathbb{D}^{1,2}$ Zhiqian Wang, ${ }^{1}$ Chang Liu, ${ }^{1}$ Qinwen Li, ${ }^{1,2}$ Jianrong Li, ${ }^{1}$ \\ and Shaojin Liu' ${ }^{1}$ \\ ${ }^{1}$ Changchun Institute of Optics Fine Mechanics and Physics, Chinese Academy of Sciences, Jilin, Changchun, China \\ ${ }^{2}$ University of Chinese Academy of Sciences, Beijing, China \\ Correspondence should be addressed to Chengwu Shen; chengwushen@163.com
}

Received 28 August 2020; Revised 29 December 2020; Accepted 29 January 2021; Published 15 February 2021

Academic Editor: Zengshun Chen

Copyright ( $\odot 2021$ Chengwu Shen et al. This is an open access article distributed under the Creative Commons Attribution License, which permits unrestricted use, distribution, and reproduction in any medium, provided the original work is properly cited.

Vehicle platform vibration (VPV) directly affects the measurement accuracy of precise measuring instrument (PMI) fixed on it. In order to reduce the influences of VPV on measurement accuracy, it is necessary to perform vibration isolation between vehicle platform and PMI. Analysis of vibration characteristics is a prerequisite for vibration isolation. However, empirical mode decomposition (EMD) and ensemble empirical mode decomposition (EEMD) reveal that there is obvious mode mixing phenomenon in the collected VPV signals. In this paper, a noise stretch ensemble empirical mode decomposition (NSEEMD) method is proposed to suppress mode mixing, and the specific operation process of NSEEMD is expounded. By NSEEMD, mode mixing of the collected platform vibration data is well suppressed, and the principal component of platform vibration can be obtained.

\section{Introduction}

A stable platform is the basis for the normal operation of precision measuring instrument (PMI). The vehicle measurement platform is greatly affected by vibration instrument such as vehicle engines and generators. The vibration can be directly transmitted to PMI and thus affects the measurement results. To ensure the measurement accuracy of PMI, a vibration isolation device is usually installed between the vehicle platform and PMI to provide a relatively stable measurement environment.

In order to obtain vibration characteristics of the vehicle platform, acceleration sensors are often used to collect the vibration information of the vehicle platform. In the information collection process, the vibration information is inevitably mixed with some interference such as environmental and circuit noises. Consequently, the collected platform vibration data must be further analyzed to extract the real vibration information of vibration sources, thus providing a reliable basis for vibration isolation design $[1,2]$.
The most important purpose of signal analysis is to clearly express the time and frequency characteristics of the signals. Traditional signal time-frequency analysis methods, such as short-time Fourier transform, Wigner-Ville distribution, and wavelet transform, are all based on the Fourier transform. These methods view the signal as a function of time or frequency as a whole and cannot accurately describe the characteristics of frequency over time. Hence, they are greatly limited by obvious defects such as poor adaptability in practical applications.

Empirical mode decomposition (EMD) method can decompose the fluctuation or change trend of different scales in the signal into a series of intrinsic mode function (IMF) sequences with actual physical significance. Since IMF comes from the signal itself, it is adaptive and has been widely used in the nonlinear vibration analysis. The Hilbert transform of IMF is called Hilbert-Huang transform (HHT), and the results can reflect the actual physical process of original data and the distribution of energy on the spatial or time scale [3]. 


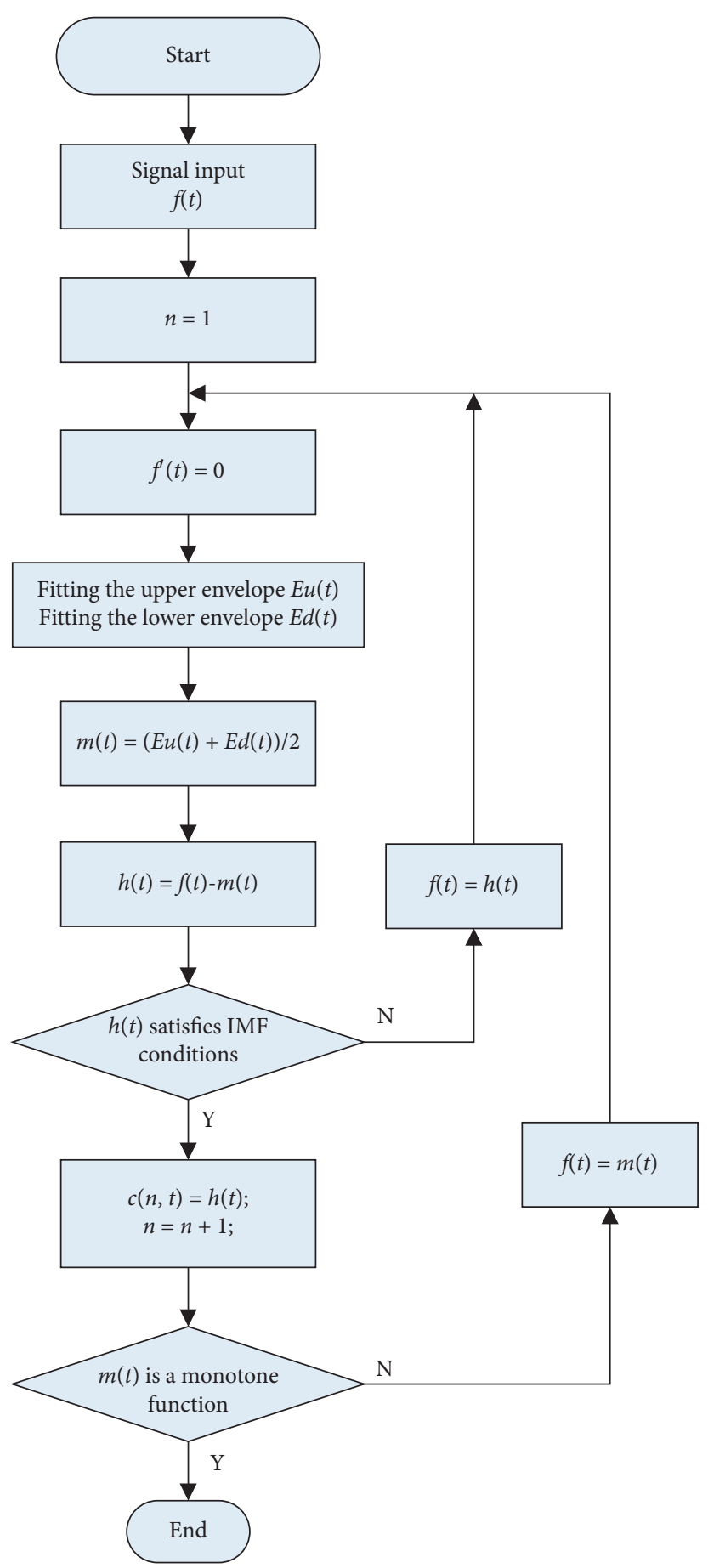

Figure 1: Flowchart of EMD. 


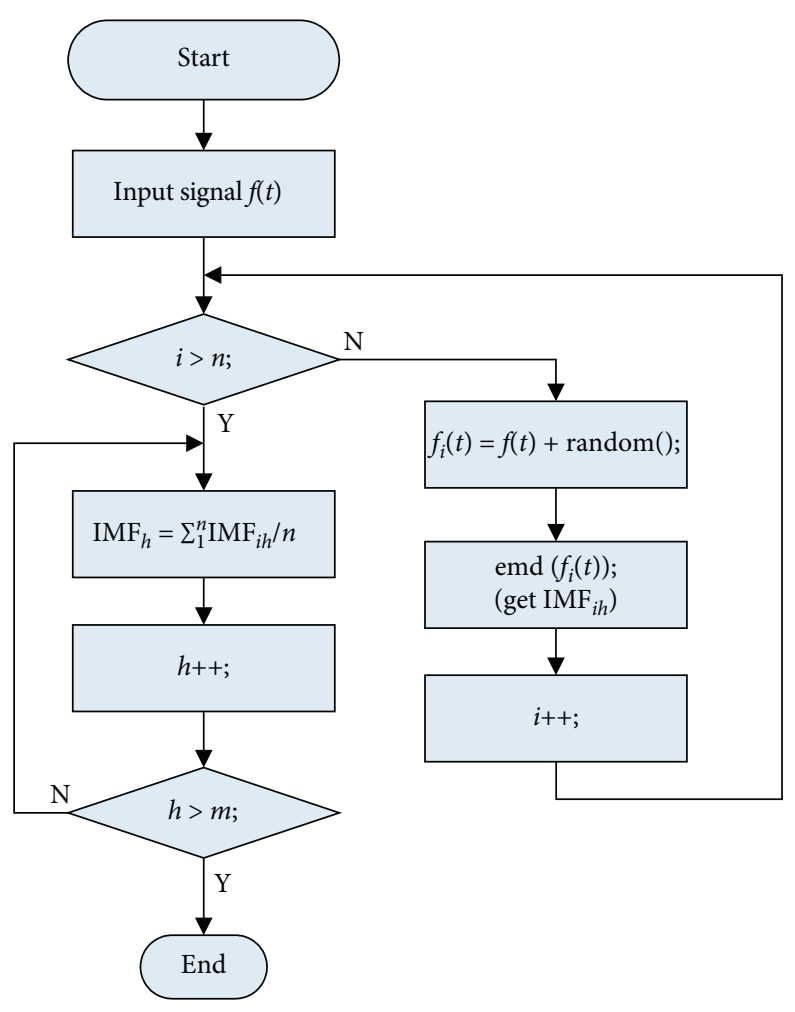

Figure 2: Flowchart of EEMD.

Ever since Huang proposed the EMD method, a large number of scholars have carried out massive research work.

Currently, the related theoretical research mainly pays attention to mathematical principles of EMD, end effect processing, mode mixing problems, and mean curve fitting. $\mathrm{Wu}$ and Huang studied the mathematical statistical characteristics of EMD based on white noise and the noiseassisted data processing method and revealed the binary filtering effect of EMD [4,5]. Qian explored the mathematical roots of HHT [6]. Wang proposed the EMD endpoint extension method of the aggregation matrix [7]. Zhou proposed a method to eliminate the end effect and false mode of EMD [8]. Based on ensemble empirical mode decomposition (EEMD), complementary ensemble empirical mode decomposition (CEEMD), and partial ensemble empirical mode decomposition (PEEMD), Huang developed a new method that combines permutation entropy and spectral substitution with ensemble EMD to solve the problem of mode mixing [9]. Zheng proposed the PEEMD method to resolve the mode mixing problem [10]. Yang used the frequency shift of Hilbert to make the signal satisfy the decomposition condition of EEMD [11]. Tang improved the fixed-point algorithm based on independent component analysis to separate the overlapping components [12]. Liu discussed the measurement and elimination of mode splitting in his study [13].

In terms of applied research, the EMD method has also been widely used in many fields such as mechanical fault diagnosis, weather forecasting, and financial market forecasting. Cheng applied the frequency family separation method to gear fault diagnosis [14]. Li applied EMD on railway wheel flat detection [15]. Li and Abdelkader applied EMD to diagnose rolling bearing faults, respectively $[16,17]$. Fang applied EMD to forecast agricultural product futures prices and Willard applied EMD to analysis of rainfall and temperature data $[18,19]$. All the above applied research has achieved good results.

In this study, we mainly focus on the methods and applications of suppressing mode mixing of EMD. The EEMD method has a certain suppression effect on mode mixing in some IMFs, but new mode mixing may appear in other IMFs. To alleviate the drawbacks of EEMD in mode mixing suppression for complex signals, the NSEEMD method is proposed, and the experimental results show that its mode mixing suppression effect is significantly better than that of EEMD.

The rest of this paper is organized as follows. Section 2 introduces the principles of EMD, HHT, and EEMD and presents mode mixing of EMD. In Section 3, the NSEEMD method is proposed and the procedures are provided. Then, in Section 4, EMD, EEMD, and NSEEMD methods are used to process specific VPV signals, and the experimental results are presented. Finally, some conclusions are drawn in Section 5 .

\section{EMD and Mode Mixing}

In this section, the principles of EMD, HHT, and EEMD are introduced, and mode mixing of EMD is presented.

2.1. Principle of EMD. The EMD is a nonlinear nonstationary signal analysis method proposed by Huang in 1998 


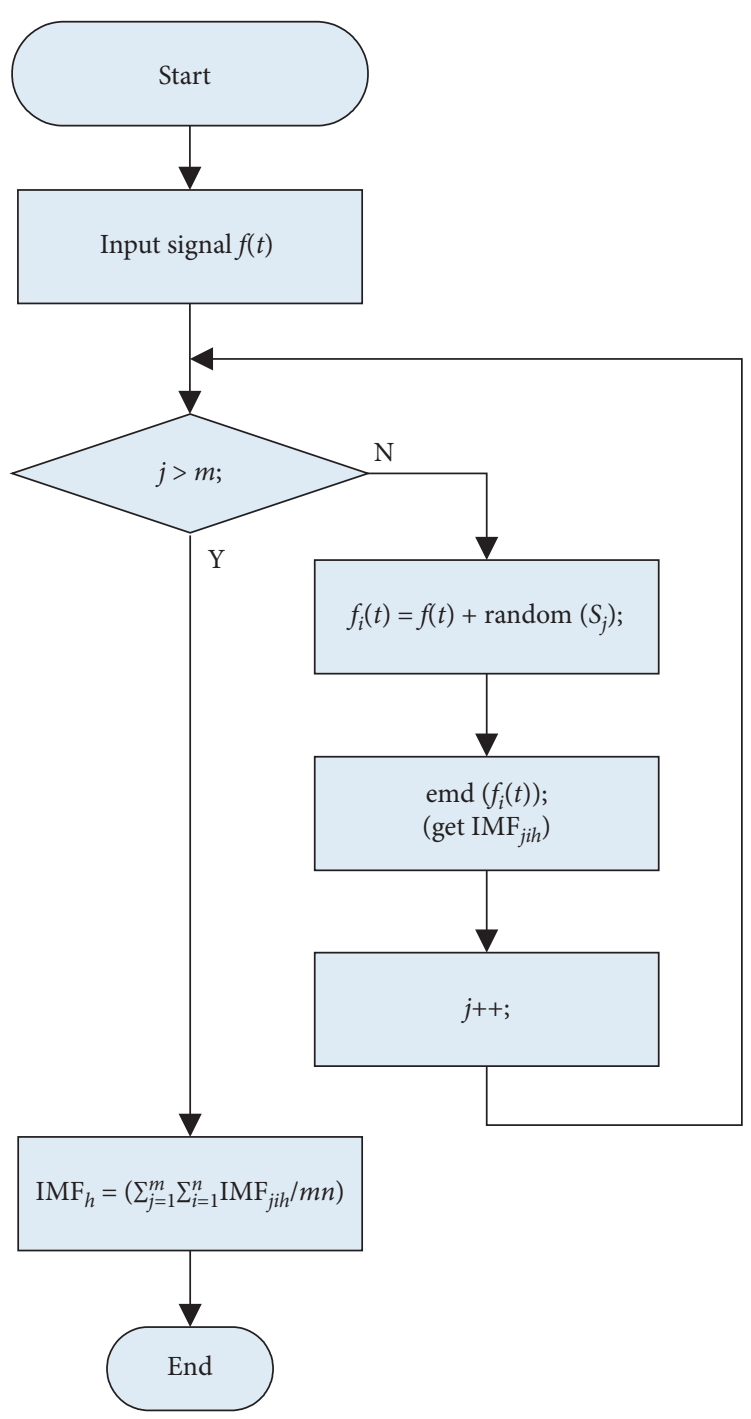

FIGURE 3: Flowchart of NSEEMD.

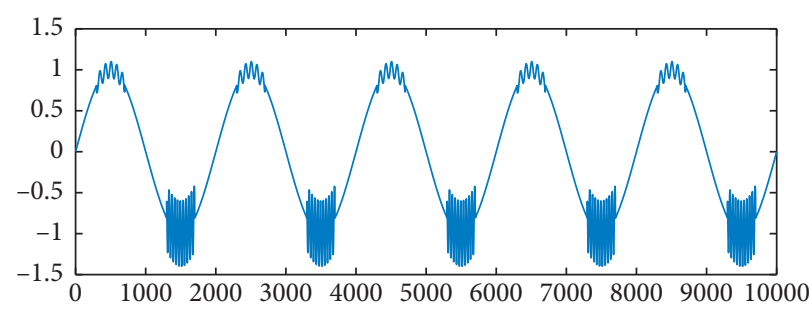

Figure 4: Simulation signal.

based on HHT. It is a powerful signal analysis technique for nonstationary and nonlinear systems. The algorithm of EMD is adaptive and data-driven, which can decompose a time series of multiple frequencies into a number of components through a sifting process in time domain. Typically, each component, referred to as an intrinsic mode function [3], follows a trend characterized by a dominant frequency, which usually varies with time. This resembles the feature of nonstationary and nonlinear systems.
Huang defines two conditions that an IMF needs to meet:

(1) Within the entire data sequence, the number of zero crossings is equal to that of maximum and minimum values or differs from that by at most 1

(2) At any point on the signal data, the average value of the upper envelope defined by the local maximum and the lower envelope defined by the minimum is 


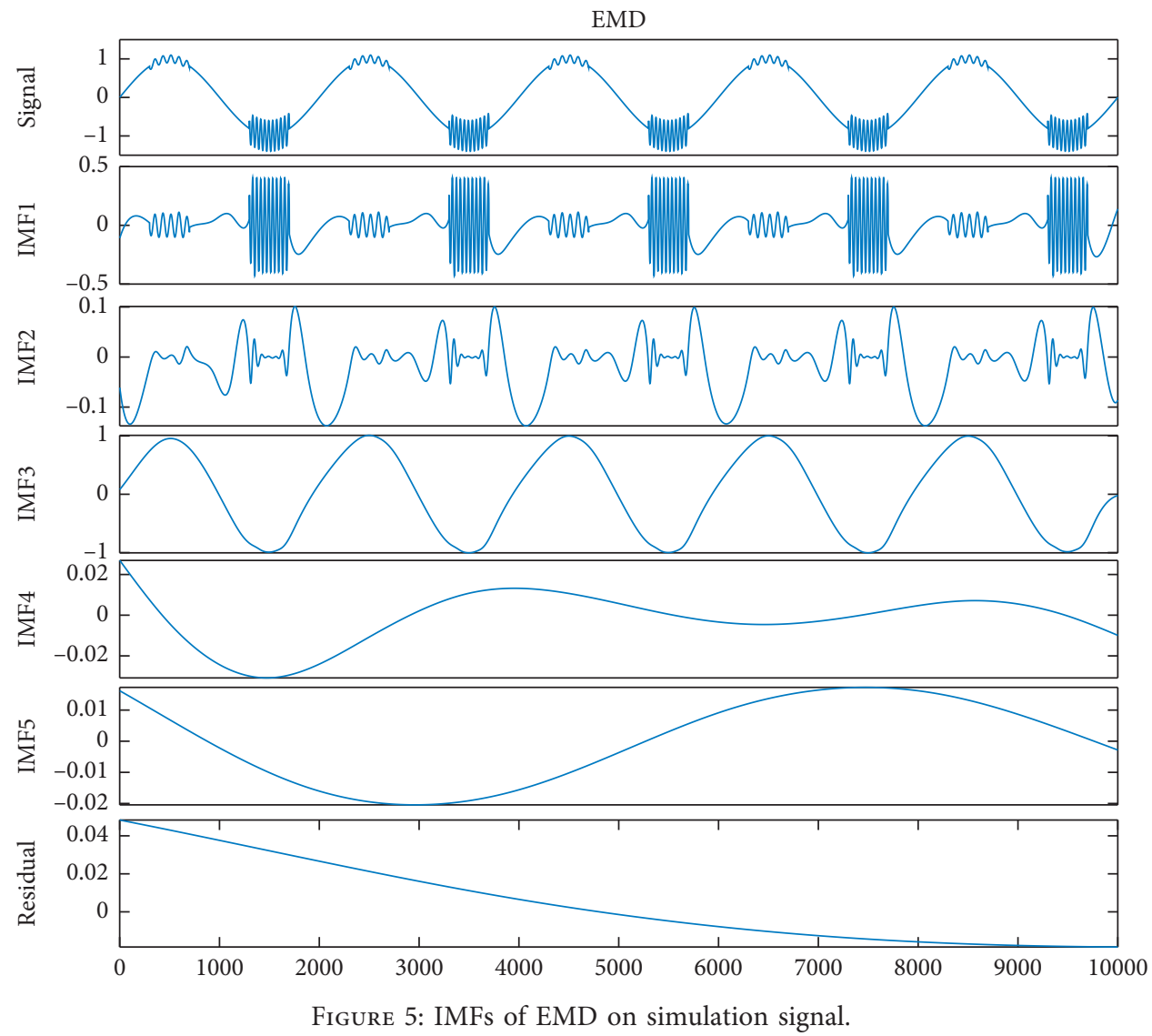

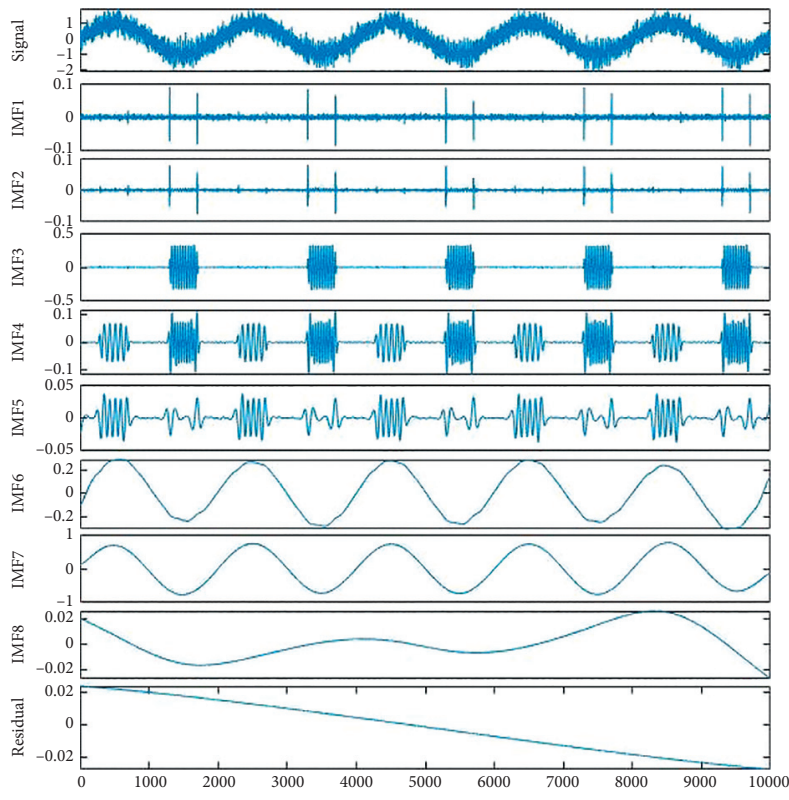

(a)

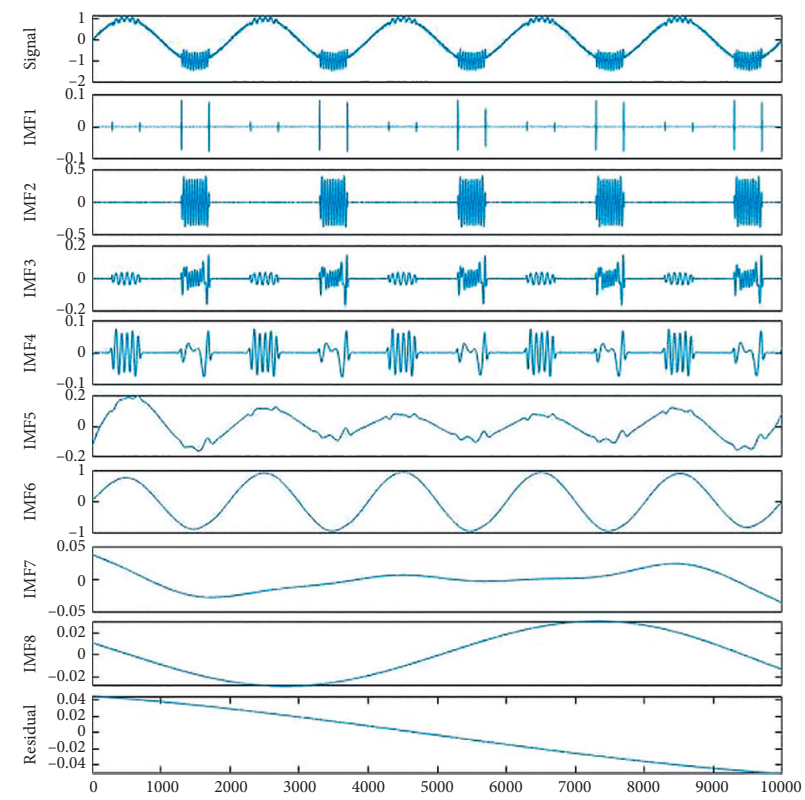

(b)

FIgURE 6: IMFs of EEMD on simulation signal. (a) EEMD with WGN in $-10 \mathrm{dBW}$. (b) EEMD with WGN in $-30 \mathrm{dBW}$. 

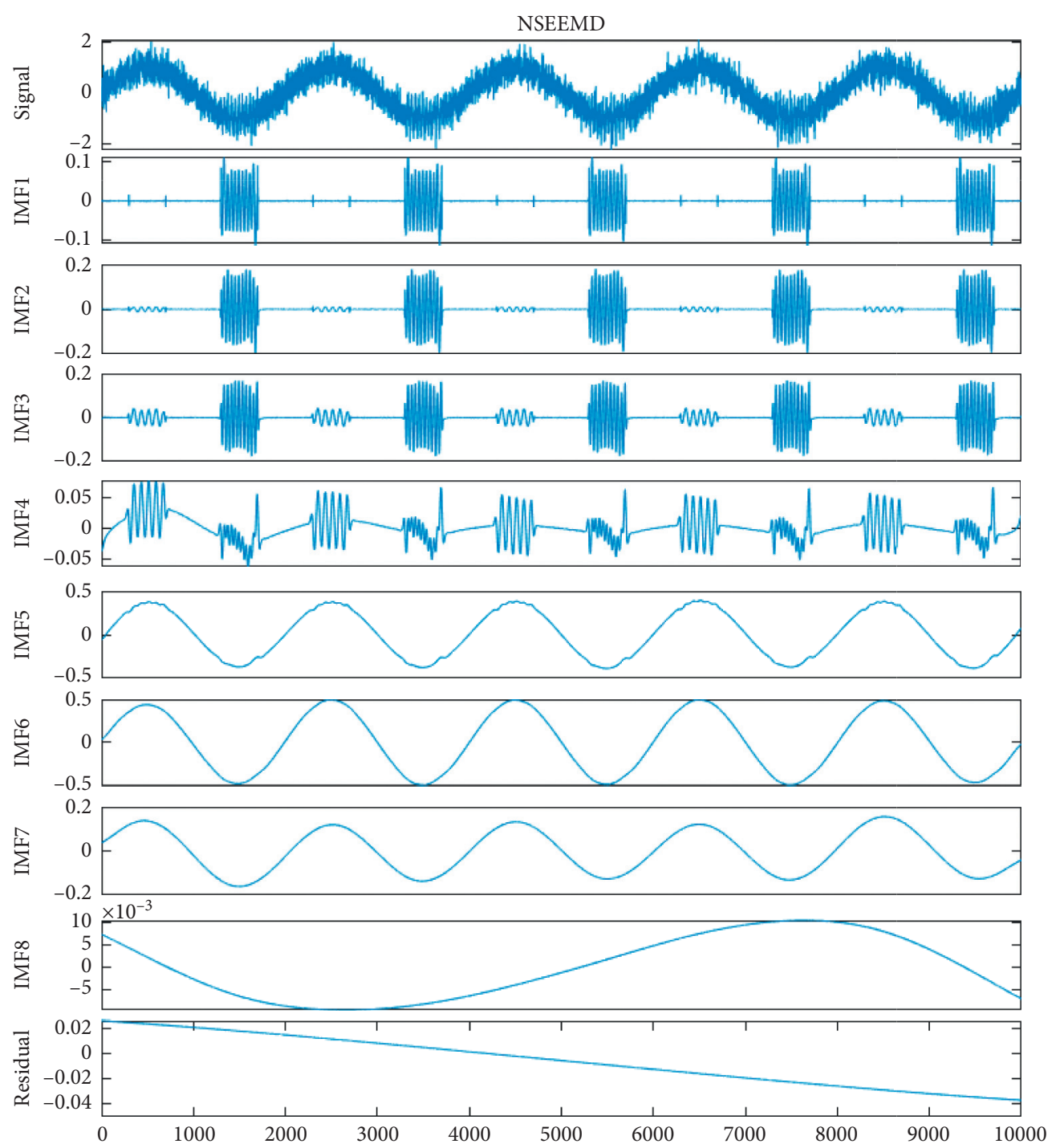

FIGURE 7: IMFs of NSEEMD on simulation signal.

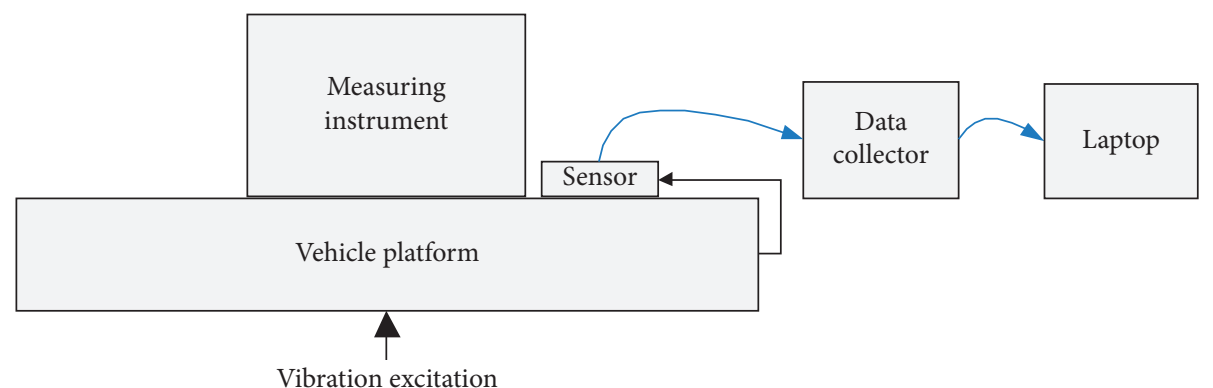

FIGURE 8: Layout of vibration information collection.

always 0 , which means the signal is locally symmetric around the time axis

These two limiting conditions make EMD similar to a narrow-band filter, and each order IMF obtained by decomposition is a set of narrow-band signals [20].

The specific procedures of EMD are shown in Figure 1.
The input signal is $f(t)$. First, determine all extreme points of $f(t)$ by $f^{\prime}(t)=0$. Then, fit the upper envelope $E_{u}(t)$ containing all maximum points and the lower envelope $E_{d}(t)$ containing all minimum points, and average the upper envelope and the lower envelope as $m(t)$. Use $h(t)$ as the signal to be decomposed if $h(t)=f(t)-m(t)$ does not 


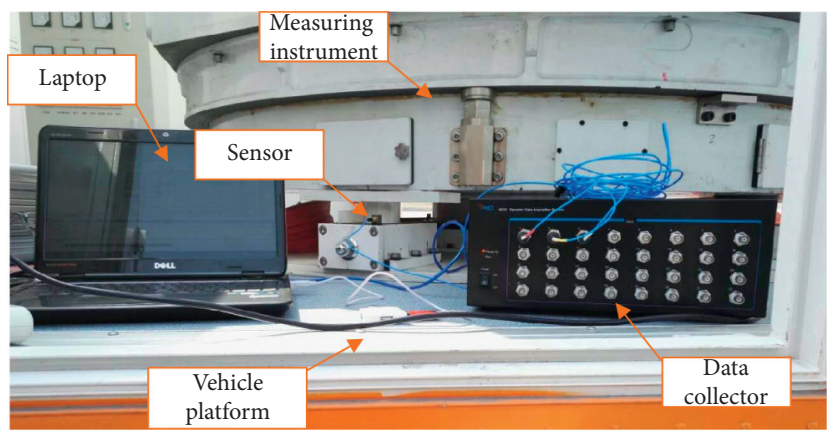

FIgURE 9: Apparatuses of vehicle platform vibration test.

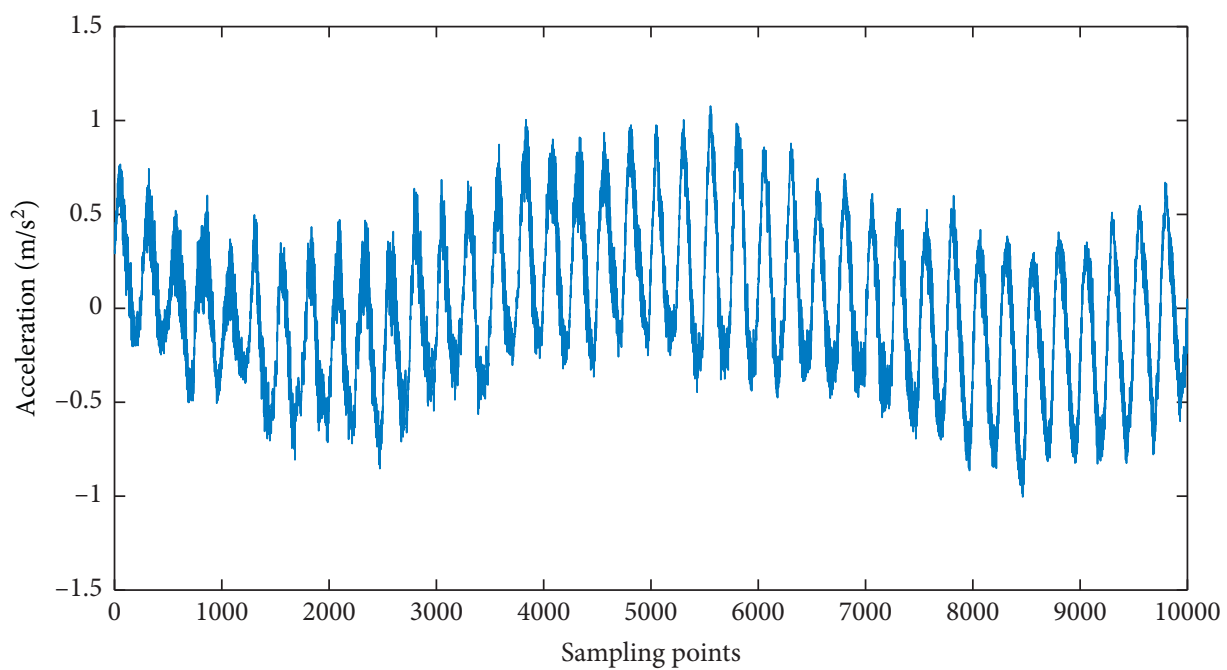

Figure 10: Sampling points of VPV.

satisfy the two IMF conditions; otherwise, $h(t)$ is the firstorder IMF. Furthermore, continue to decompose the residual $m(t)$ obtained by decomposition to obtain higher-order IMFs. The EMD ends until the residual terms obtained by decomposition are monotone functions.

There is a physical mapping relationship between a series of IMFs obtained by EMD and the inherent mode function of the system. The IMFs of EMD represent the components of the signal with different frequencies from high to low, and the residual term represents the average trend in the signal. The frequency components contained in each order IMF frequency band are different. In the same IMF component, the instantaneous frequencies at different time points are also different. This local time distribution of different frequency components varies with the signal itself [3].

2.2. Algorithm of HHT. The following Hilbert Transform is performed on each IMF function $h_{i}(t)$ :

$$
\widehat{h}_{i}(t)=H\left[h_{i}(t)\right]=\frac{1}{\pi} \int_{-\infty}^{\infty} \frac{h_{i}(t)}{t-\tau} \mathrm{d} \tau .
$$

Construct analytical signal:

$$
z_{i}(t)=h_{i}(t)+j \widehat{h}_{i}(t)=a_{i}(t) e^{j \varphi_{i}(t)} .
$$

Get the instantaneous amplitude function:

$$
a_{i}(t)=\sqrt{h_{i}^{2}(t)+\widehat{h}_{i}^{2}(t)} .
$$

The function of the instantaneous phase is

$$
\varphi_{i}(t)=\arctan \frac{\widehat{h}_{i}(t)}{h_{i}(t)}
$$

The function of the instantaneous frequency is

$$
f_{i}(t)=\frac{1}{2 \pi} \omega_{i}(t)=\frac{1}{2 \pi} \times \frac{\mathrm{d} \varphi_{i}(t)}{\mathrm{d} t} .
$$

The function of Hilbert spectrum is

$$
H(\omega, t)=\operatorname{RP} \sum_{i=1}^{n} a_{i}(t) e^{j\left(\int \omega_{i}(t) \mathrm{d} t\right) .}
$$

The function of Hilbert marginal spectrum is

$$
h(\omega)=\int_{0}^{T} H(\omega, t) \mathrm{d} t .
$$

The Hilbert spectrum shows the joint distribution of signal amplitude with time and frequency. The Hilbert marginal spectrum reflects the variation of signal amplitude 


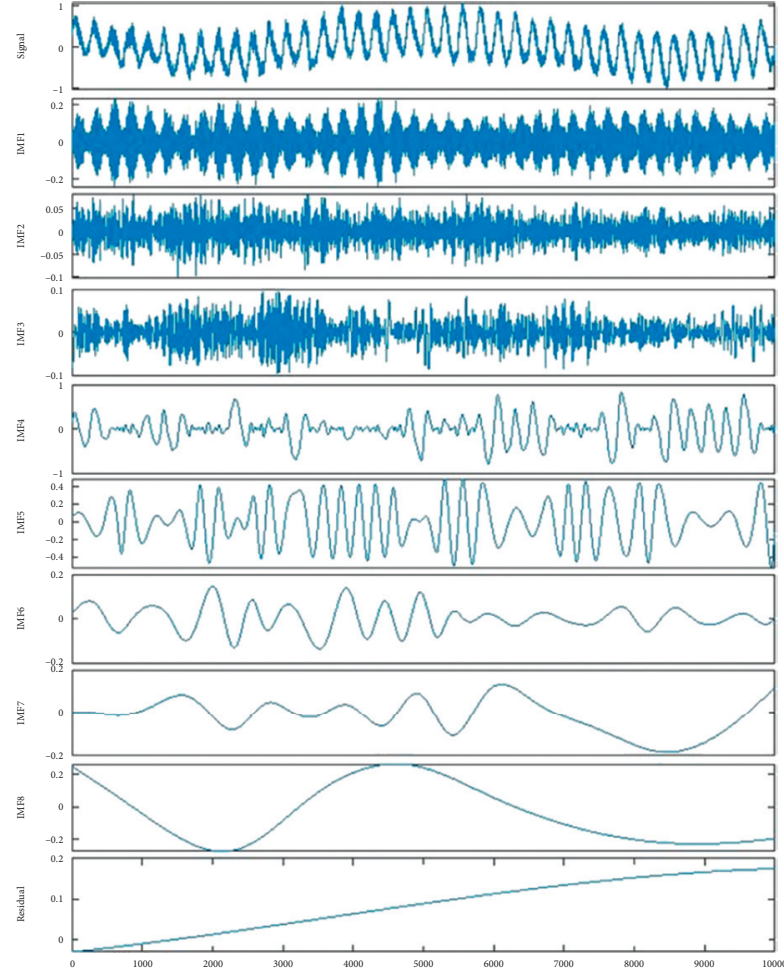

(a)

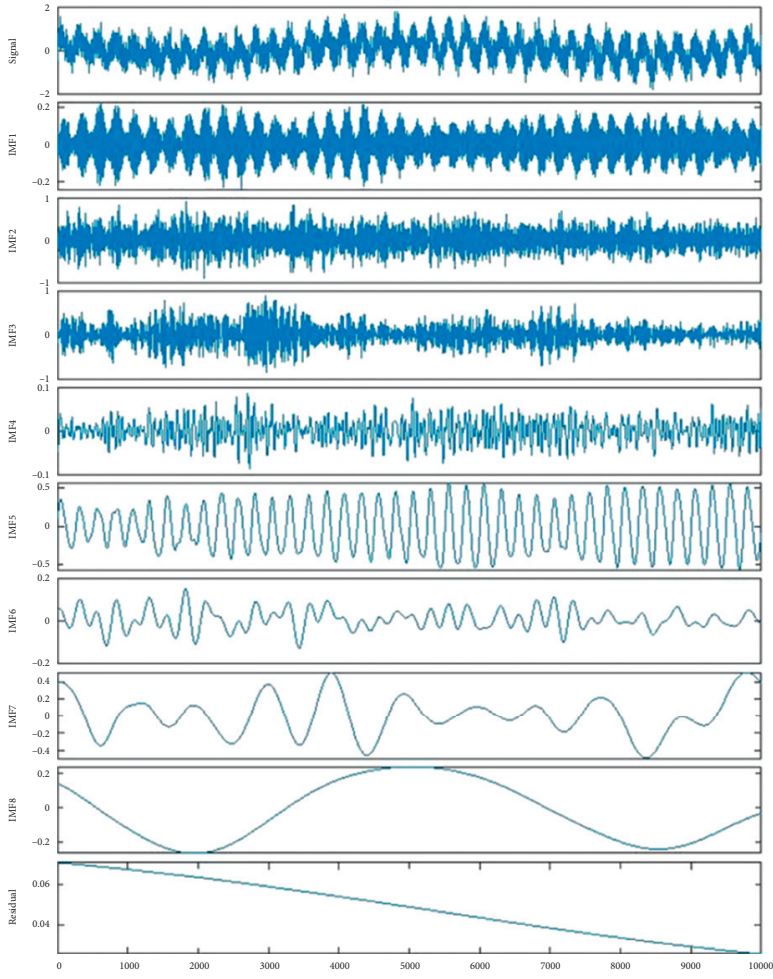

(c)

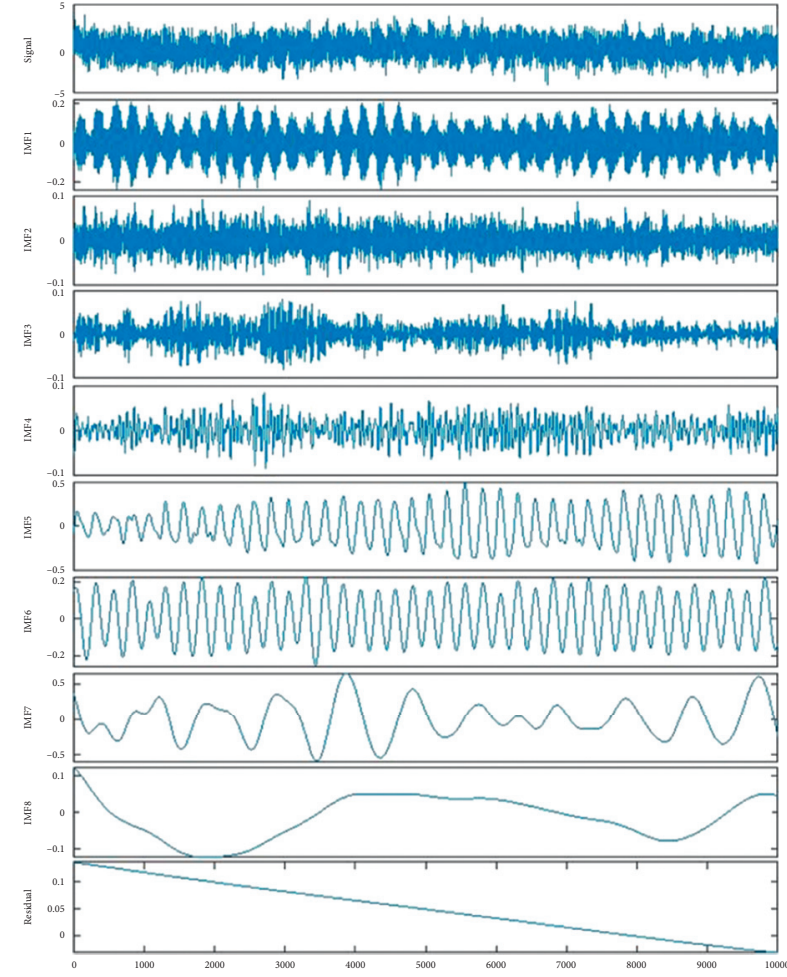

(b)

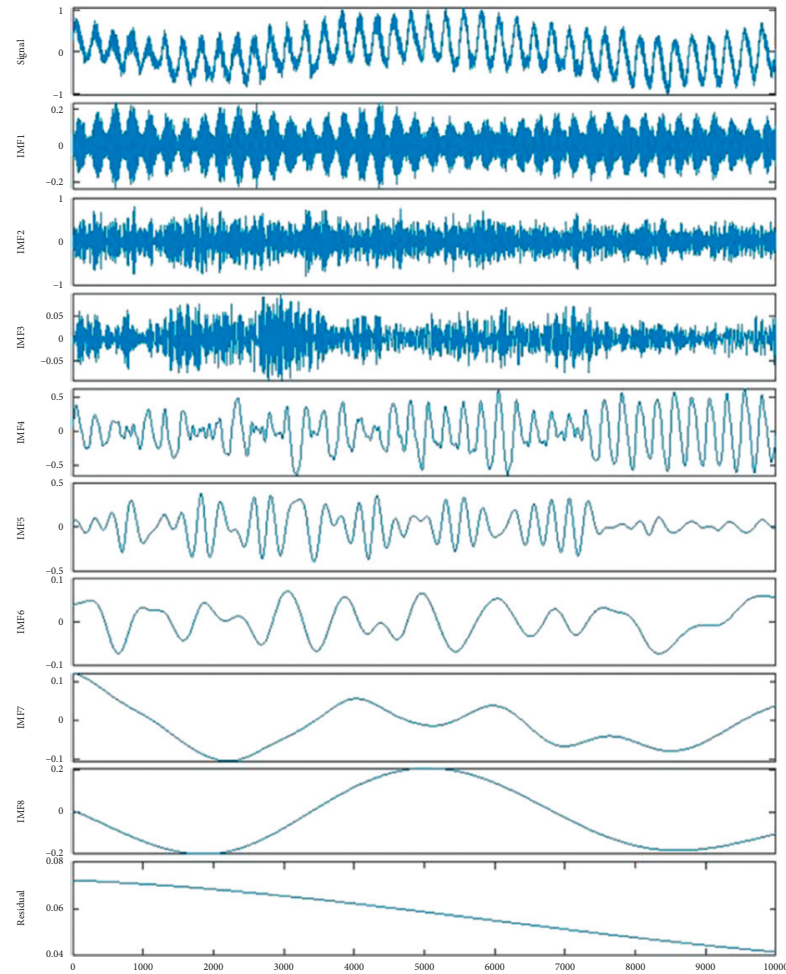

(d)

Figure 11: Continued. 


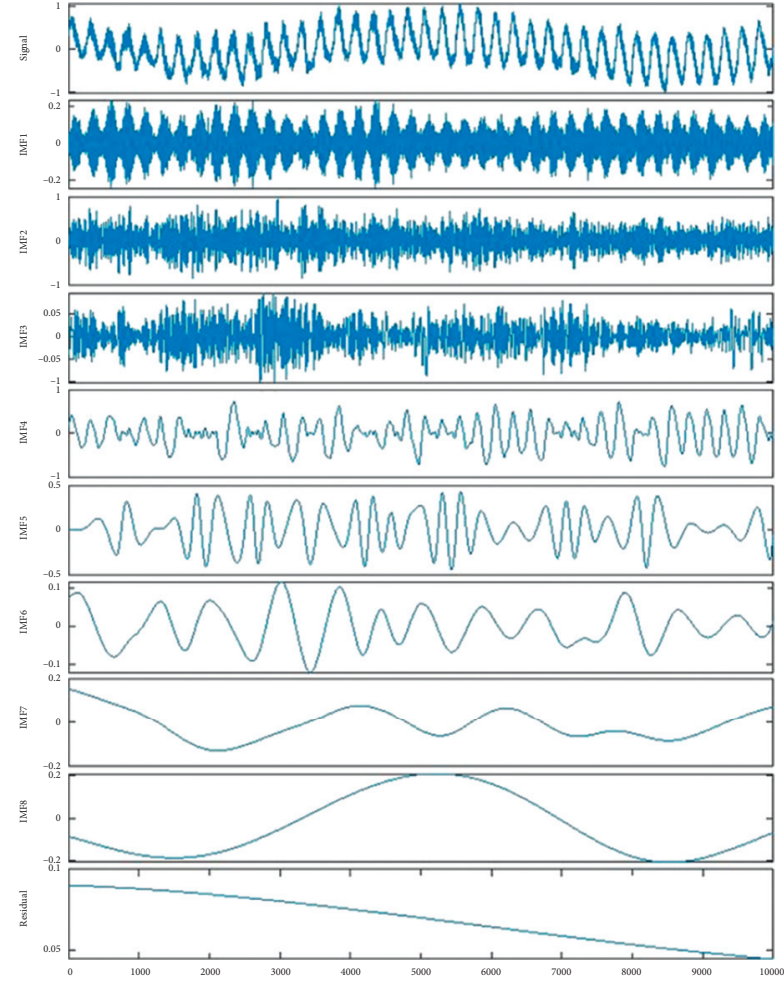

(e)

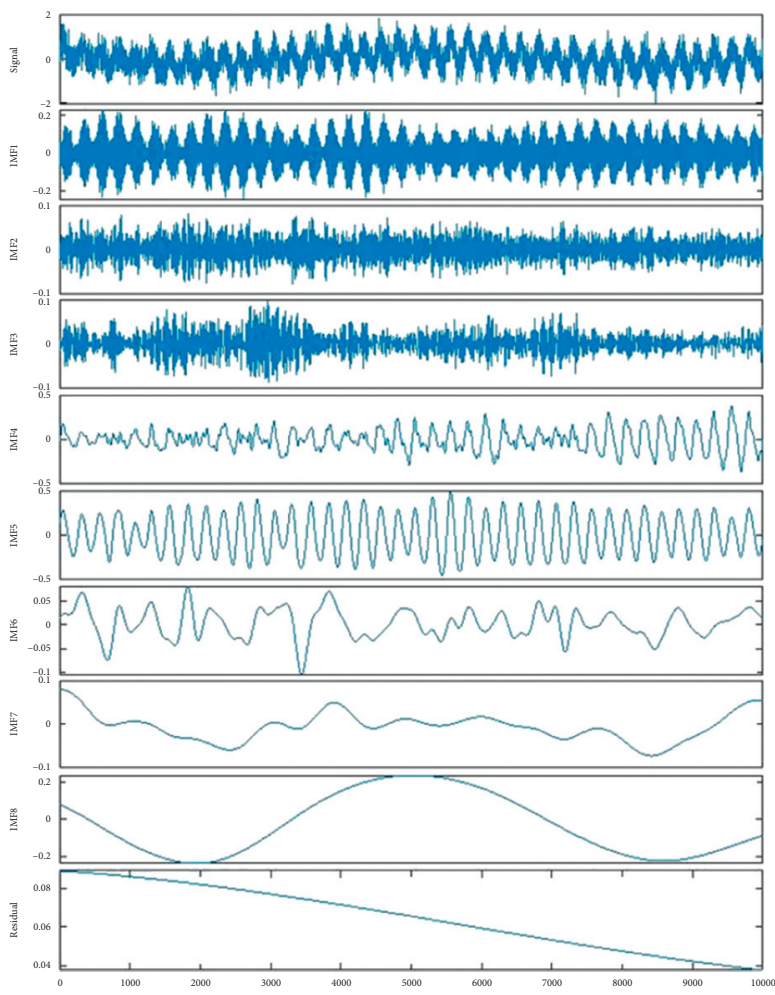

(f)

FIGURE 11: IMFs of VPV data processing by different methods. (a) IMFs obtained by EMD. (b) IMFs obtained by EEMD with WGN in $0 \mathrm{dBW}$. (c) IMFs obtained by EEMD with WGN in $-10 \mathrm{dBW}$. (d) IMFs obtained by EEMD with WGN in $-30 \mathrm{dBW}$. (e) IMFs obtained by EEMD with WGN in $-50 \mathrm{dBW}$. (f) IMFs obtained by NSEEMD proposed in this study.

with frequency. The Hilbert marginal spectrum is equivalent to the Fourier spectrum, but has higher resolution.

The essence of EMD is a binary filter. IMF1, the first component, represents the highest frequency segment in the signal. The relationship between the center frequency the $i$ th IMF and the center frequency $f_{i+1}$ of the $(i+1)$ th IMF is

$$
f_{i+1}=\frac{f_{i}}{2} \text {. }
$$

The EMD has the characteristics of frequency determination and adaptation. The determination of the center frequency of IMF depends on the signal itself and has nothing to do with human factors. When the center frequency $f_{1}$ of the first IMF is determined, the center frequencies of subsequent IMFs are all related to $f_{1}$. When there is no frequency component of a certain frequency band in the signal, the center frequency of the next IMF is determined by the actual frequency lower than that frequency band [20].

2.3. Mode Mixing and EEMD. The binary filtering characteristic of EMD can decompose the signal into a series of IMF components with different center frequencies, and the center frequency of IMF is strictly kept at $1 / 2$ of the previous one. However, when one or some signal components in original data are nonuniformly distributed on time and frequency scales, some IMFs may contain two frequencies that are not in the same frequency band. Mode mixing is defined based on this chaotic phenomenon caused by the loss of time scale during the EMD process. It is noticed that such mode mixing phenomenon makes each order IMF lose its physical meaning.

In order to suppress mode mixing, $\mathrm{Wu}$ and Huang proposed the EEMD method. The specific idea of EEMD is to add an evenly distributed white noise sequence to the test data $f(t)$ and obtain a new data sequence $f_{1}(t)$. First, perform EMD on $f_{1}(t)$ to obtain an $\mathrm{IMF}_{i h} \mathrm{IMF}_{1 h}$. Then, repeat the above process and add different white noises to the test data each time, thus obtaining a series of $\mathrm{IMF}_{i n}$. Finally, average IMFs of the same order as the final result through the following mathematical expression:

$$
\mathrm{IMF}_{h}=\frac{\left(\sum_{i=1}^{n} \mathrm{IMF}_{i h}\right)}{n} .
$$

The procedures of EEMD are shown in Figure 2:

\section{NSEEMD Method and Simulation}

During VPV signal processing, it is found that the signal is affected by multiple vibration sources and circuit noise. As a result, the obtained platform vibration data are complicated, and the vibration frequencies are distributed in a wide range. 


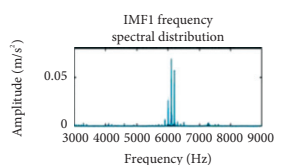

Frequency $(\mathrm{Hz})$

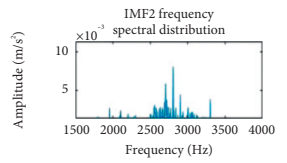

IMF3 frequency

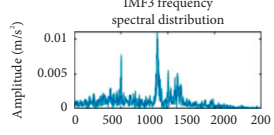

Frequency (Hz)

IMF4 frequency
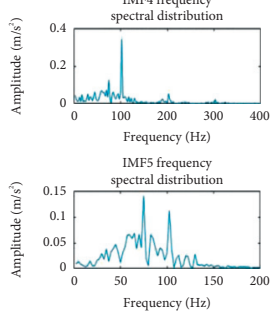

Frequency $(\mathrm{Hz})$

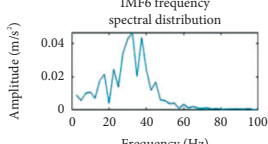

Frequency $(\mathrm{Hz})$

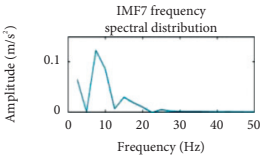

IMF8 frequency

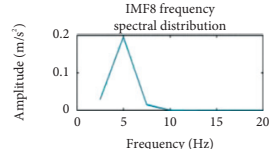

(a)

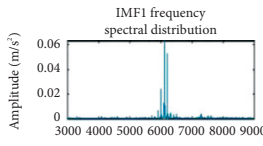

Frequency $(\mathrm{Hz})$

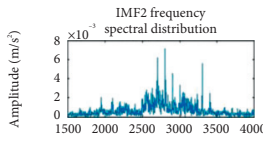

2500250030003500
Frequency $(\mathrm{Hz})$

IMF3 frequency

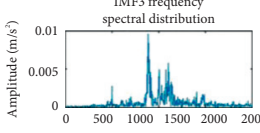

Frequency $(\mathrm{Hz})$

IMF4 frequency
spectral distribution
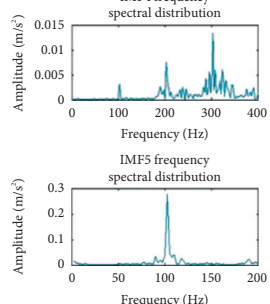

Frequency (H

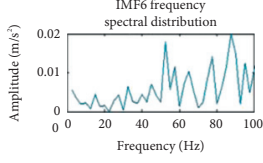

IMF7 frequency

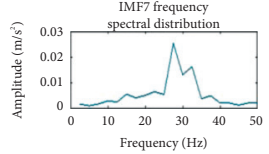

IMF8 frequency

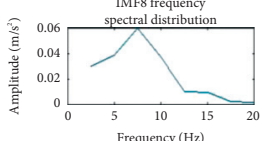

(b)

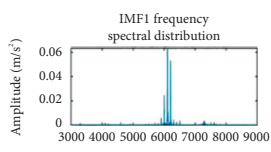

Frequency $(\mathrm{Hz})$

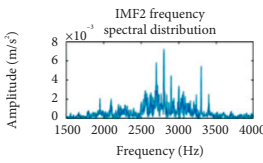

IMF3 frequency

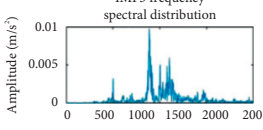

Frequency $(\mathrm{Hz})$

IMF4 frequency
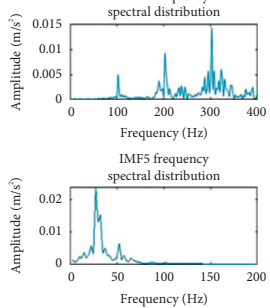

IMF6 frequency
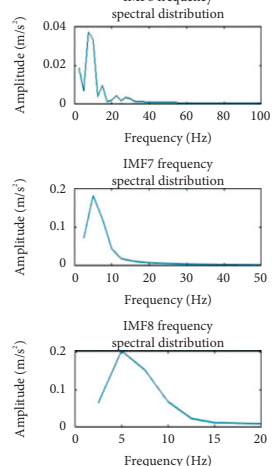

(c)

Figure 12: Continued. 


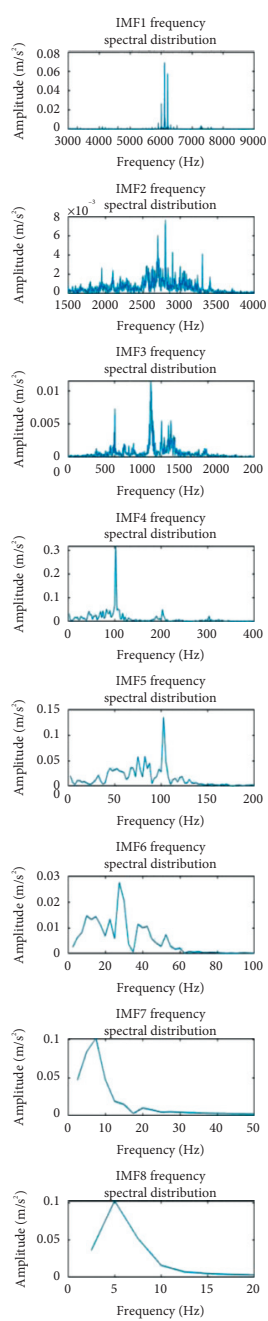

(d)

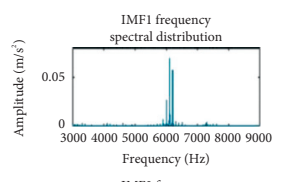

IMF2 frequency

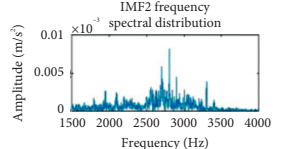

Frequency $(\mathrm{Hz})$

IMF3 frequency
spectral distribution
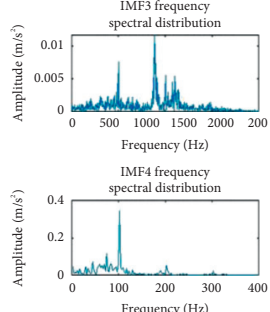

IMF5 frequency

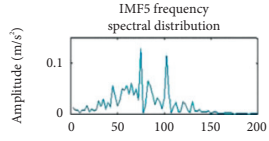

Frequency $(\mathrm{Hz})$

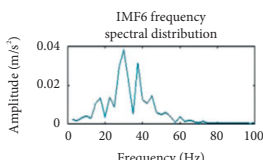

IMF7 frequs

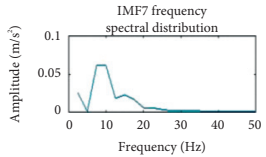

IMF8 frequency

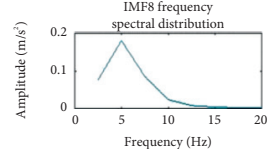

(e)

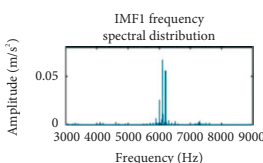

Frequency (Hz)
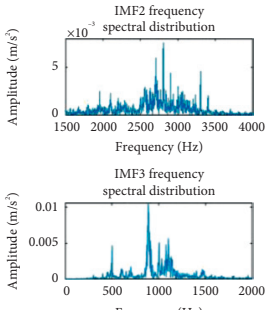

Frequency $(\mathrm{Hz})$

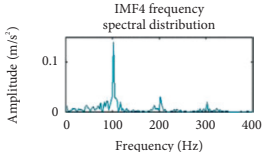

IMF5 frequency

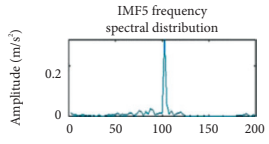

Frequency $\left(\mathrm{H}_{z}\right)$

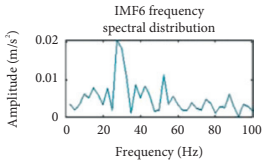

Frequency (Hz)
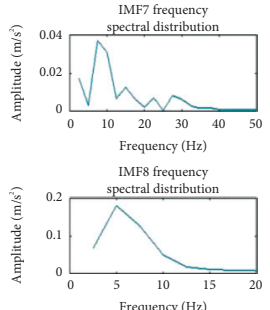

(f)

FIGURE 12: Hilbert marginal spectrum of IMFs by different methods. (a) EMD. (b) EEMD with WGN in 0 dBW. (c) EEMD with WGN in $-10 \mathrm{dBW}$. (d) EEMD with WGN in $-30 \mathrm{dBW}$. (e) EEMD with WGN in $-50 \mathrm{dBW}$. (f) NSEEMD proposed in this study.

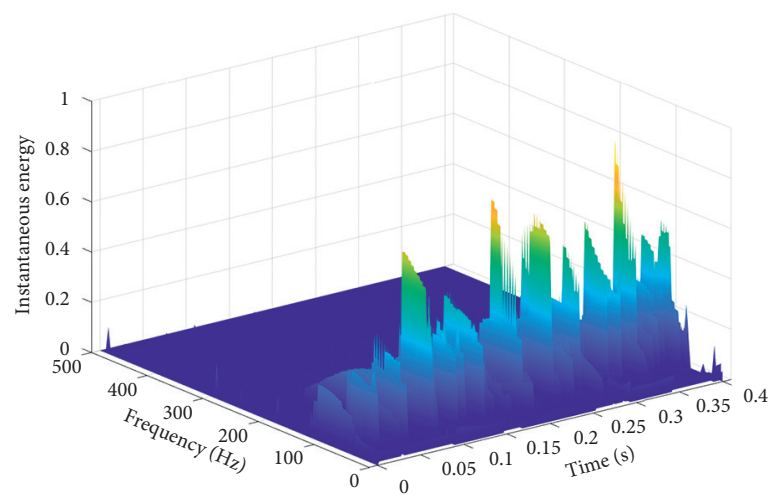

Figure 13: Hilbert spectrum of $0-500 \mathrm{~Hz}$.

When the vibration data are processed by EMD, there is obvious mode mixing in some IMFs. When the data are processed by EEMD, mode mixing in some IMFs can be suppressed to a certain extent. It is also found in the experiment that mode mixing suppression is affected by the power spectral density (PSD) of white noise. However, white noise with a certain PSD only suppresses mode mixing in a certain frequency band. In order to achieve mode mixing 
suppression in a wider frequency range, this paper proposes an NSEEMD method, and the specific procedures of NSEEMD are as follows:

(a) Add a white Gaussian noise (WGN) sequence $g_{1}(t)$ in a PSD $S_{1}$ to $f(t)$, obtain a new data sequence $f_{1}(t)$, perform EMD on $f_{1}(t)$, obtain an IMF sequence $\mathrm{IMF}_{11 h}$, and repeat the above process to obtain a series of $\mathrm{IMF}_{1 i h}$.

(b) Repeat (a). Add a WGN sequence $g_{1}(t)$ in PSD $S_{j}$ to $f(t)$, and obtain a series of $\operatorname{IMF}_{j i h}$.

(c) Average IMFs of the same order obtained by each EEMD as the final result through the following mathematical expression:

$$
\mathrm{IMF}_{h}=\frac{\sum_{j=1}^{m} \sum_{i=1}^{n} \mathrm{IMF}_{j i h}}{m n} .
$$

The procedures of NSEEMD are shown in Figure 3:

Mode mixing is caused by the loss of time scale in the EMD process. Add the white noise signal $n_{i}(t)$ to the signal $f(t)$ to form a new signal $S_{i}(t)$ as

$$
\frac{1}{m} \sum_{i=1}^{m} S_{i}(t)=\frac{1}{m} \sum_{i=1}^{m} f(t)+\frac{1}{m} \sum_{i=1}^{m} n_{i}(t) .
$$

Here, $S_{i}(t)$ is continuous on the time scale.

The statistical characteristic of the white noise shows

$$
\frac{1}{m} \sum_{i=1}^{m} n_{i}(t)=0 \text {. }
$$

Thus, the IMF of $f(t)$ can be obtained by averaging the IMFs of all orders of $S_{i}(t)$.

Here, we construct a sinusoidal signal with high-frequency intermittent disturbances, as shown in Figure 4. The main frequency signal is $\sin (\pi i / 1000)$, and the disturbance signals are $0.1 \sin (\pi i / 400)$ and $0.4 \sin (\pi i / 20)$.

We perform EMD on the above signals to obtain IMFs, as shown in Figure 5. It can be seen that IMF1 has mode mixing and that the modes of the two added disturbance signals are not separated.

We perform EEMD on the simulation signal with GWN in $-10 \mathrm{dBW}$ and $-30 \mathrm{dBW}$, respectively, to obtain IMFs, as shown in Figure 6. It can be seen that mode mixing is suppressed to a certain extent.

We perform NSEEMD on the simulation signal to obtain IMFs, as shown in Figure 7. It can be seen that the modes of the two added disturbance signals are well separated.

\section{Vehicle Platform Vibration Process and Result Analysis}

In order to obtain the vibration information of the vehicle platform, a sensor is fixed near the location where the measuring instrument is connected to the vehicle platform. The sensor converts the vibration information into electrical signals, which are collected by a data collector. The data collector then transmits the vibration information data to a laptop, and the laptop stores the vibration information data. Figure 8 shows the layout of vibration information collection.

In this paper, the vehicle platform vibration data are collected by the apparatuses shown in Figure 9. A piezoelectric acceleration sensor 356A26PCB produced by PCB Company is used as the sensor, and a YMC-9800 dynamic data collector produced by YMK Measurement and Control Technology Co., Ltd. is employed for data acquisition. The connection position between the vehicle platform and the measuring instrument is on the vibration transmission path, and the acceleration sensor is placed in the sensitive position. The sampling frequency is set as $25 \mathrm{kHz}$.

The number of sampling points is set as 10,000 , and the original sampling data of VPV are shown in Figure 10.

The EMD, EEMD, and NSEEMD are, respectively, performed on the sampling data, and the obtained IMFs are shown in Figure 11.

The HHT is performed on IMFs, and Figure 12 presents the vibration Hilbert marginal spectrum (HMS) of IMFs.

From Figures 11 and 12, we can clearly draw the following conclusions:

(1) When the EMD method is used to process the vibration data, there is obvious mode mixing in IMF3 and IMF5.

(2) When the EEMD method is used to process the vibration data, if the power spectral density of WGN is $-10 \mathrm{dBW}$, mode mixing in IMF3 and IMF5 can be suppressed, but new mode mixing appears in IMF4 and IMF6. If the power spectral density of WGN is $-30 \mathrm{dBW}$, mode mixing in IMF5 is suppressed, but mode mixing in IMF3 is not suppressed. If the power spectral density of WGN is $-50 \mathrm{dBW}$, the power spectral density is too low, and the suppression effect on mode mixing is weak.

(3) When the NSEEMD method proposed in this study is used to process the vibration data, mode mixing in IMF3 and IMF5 can be well suppressed, and there is no new mode mixing.

From mode decomposition, it can be seen that the vibration energy of the vehicle platform is mainly concentrated at $105 \mathrm{~Hz}$. Figures 13 presents the Hilbert spectrum of IMFs obtained by NSEEMD in the range of $0-500 \mathrm{~Hz}$, which clearly displays the distribution of frequency and energy in time domain.

\section{Conclusion}

In summary, there is obvious mode mixing phenomenon in the collected VPV signals by EMD and EEMD, and NSEEMD is proposed to suppress mode mixing in IMFs.

Mode decomposition of the VPV data by EMD can result in obvious mode mixing of the obtained IMFs. EEMD can only suppress partial mode mixing of complex signals, but may create new mode mixing. Experimental results show that the mode mixing suppression capability of EEMD is related to the power spectral density of the added WGN, 
which determines the frequency band and suppression ability of mode mixing that it can suppress.

Compared with EMD and EEMD, NSEEMD can effectively suppress mode mixing with no new mode mixing and well maintain the adaptive characteristics of EMD. By separating the signals of each frequency of the VPV data, the obtained IMFs represent the actual vibration information of the vehicle platform, and the vibration frequency distribution after mode decomposition can improve the design of the vehicle platform.

\section{Data Availability}

No data were used to support the findings of the study.

\section{Conflicts of Interest}

The authors declare that they have no conflicts of interest.

\section{Acknowledgments}

This work was supported in part by the Jilin Province Key Scientific and Technological Projects of China (20190302086GX) and Jilin Province Key Scientific and Technological Projects of China (20200403067SF).

\section{References}

[1] M. Li and J. Luo, "Wavelet denoising of vehicle platform vibration signal basedon threshold neural network," Shock and Vibration, vol. 79, 2017.

[2] M. Li and J. Luo, "Wavelet Denoising of vehicle platform vibration signals based on wavelet analysis," Journal of Northeast Normal University (Natural Science Edition), vol. 49, no. 2, pp. 100-105, 2017.

[3] N. E. Huang, Z. Shen, S. R. Long et al., "The empirical mode decomposition and the Hilbert spectrum for nonlinear and non-stationary time series analysis," Proceedings of the Royal Society of London. Series A: Mathematical, Physical and Engineering Sciences, vol. 454, 1998.

[4] Z. Wu and N. E. Huang, "A study of the characteristics of white noise using the empirical mode decomposition method," Proceedings of the Royal Society of London. Series A: Mathematical, Physical and Engineering Sciences, vol. 460, no. 2046, pp. 1597-1611, 2004.

[5] Z. H. Wu and N. E. Huang, "Ensemble empirical mode decomposition: a noise assisted data analysis method," Advances in Adaptive Data Analysis, vol. 1, no. 1, pp. 1-41, 2008.

[6] T. Qian, "Mono-components for decomposition of signals," Advances in Adaptive Data Analysis, vol. 29, no. 10, pp. 1187-1198, 2006.

[7] W. Xin and W. Qian, "Improved Hilbert-Huang transform and its application," Journal of Electronic Measurement and Instrumentation, vol. 28, no. 12, pp. 1369-1375, 2014.

[8] X. Zhou, W. Liu, Z. Jiang et al., "An improved hilbert-huang transform method and its application," Advanced Engineering Sciences, vol. 49, no. 4, pp. 196-204, 2017.

[9] S. Huang, X. Wang, C. Li, and C. Kang, "Data decomposition method combining permutation entropy and spectral substitution with ensemble empirical mode decomposition," Measurement, vol. 139, pp. 438-453, 2019.

[10] J. Zheng, J. Cheng, and Yu Yang, "Partly ensemble empirical mode decomposition: an improved noise-assisted method for eliminating mode mixing," Signal Processing, vol. 96, no. 2, pp. 362-374, 2014.

[11] Z. Yang, L. Yang, and C. Qing, "An oblique-extrema-based approach for empirical mode decomposition,” Digital Signal Processing, vol. 20, no. 3, pp. 699-714, 2010.

[12] B. Tang, S. Dong, and T. Song, "Method for eliminating mode mixing of empirical mode decomposition based on the revised blind source separation," Signal Processing, vol. 92, no. 1, pp. 248-258, 2012.

[13] B. Liu, P. Zheng, Q. Dai, and Z. Zhou, “The measurement and elimination of mode splitting: from the perspective of the partly ensemble empirical mode decomposition," Complexity, vol. 2018, pp. 1-10, 2018.

[14] J. Cheng, D. Yu, J. Tang, and Y. Yang, “Application of frequency family separation method based upon EMD and local Hilbert energy spectrum method to gear fault diagnosis," Mechanism and Machine Theory, vol. 43, no. 6, pp. 712-723, 2008.

[15] Y. Li, J. Liu, and Y. Wang, "Railway wheel flat detection based on improved empirical mode decomposition," Shock and Vibration, vol. 43, 2016.

[16] R. Li, C. Ran, B. Zhang et al., "Rolling bearings fault diagnosis based on improved complete ensemble empirical mode decomposition with adaptive noise, nonlinear entropy, and ensemble SVM," Applied Sciences, vol. 10, no. 16, 2020.

[17] R. Abdelkader, A. Kaddour, A. Bendiabdellah, and Z. Derouiche, "Rolling bearing fault diagnosis based on an improved denoising method using the complete ensemble empirical mode decomposition and the optimized thresholding operation," IEEE Sensors Journal, vol. 18, no. 17, pp. 7166-7172, 2018.

[18] Y. Fang, B. Guan, S. Wu, and S. Heravi, "Optimal forecast combination based on ensemble empirical mode decomposition for agricultural commodity futures prices," Journal of Forecasting, vol. 39, no. 6, pp. 877-886, 2020.

[19] Z. Willard, K. Symala, and S. Venkataraman, "Analysis of rainfall and temperature data using ensemble empirical mode decomposition," Data Science Journal, vol. 18, no. 1, pp. 4657, 2019.

[20] Y. Yang and Y. Wu, "Theory of Empirical Mode Decomposition" in Application of Empirical Mode Decomposition in Vibration Analysis," National Defense Industry Press, vol. 18, pp. 17-24, 2013. 